\title{
Quantitative Longitudinal Imaging of Vascular Inflammation and Treatment by Ezetimibe in apoE Mice by FMT Using New Optical Imaging Biomarkers of Cathepsin Activity and $\alpha_{v} \beta_{3}$ Integrin
}

\author{
Shu-An Lin, ${ }^{1}$ Manishkumar Patel, ${ }^{1}$ Donna Suresch, ${ }^{1}$ Brett Connolly, ${ }^{1}$ \\ Bagna Bao, ${ }^{2}$ Kevin Groves, ${ }^{2}$ Milind Rajopadhye, ${ }^{2}$ Jeffrey D. Peterson, ${ }^{2}$ \\ Michael Klimas, ${ }^{1}$ Cyrille Sur, ${ }^{1}$ and Bohumil Bednar ${ }^{1}$ \\ ${ }^{1}$ Department of Imaging, Merck Research Laboratories, 770 Sumneytown Pike, WP44C-2, West Point, PA 19486, USA \\ 2 Department of Applied Biology, Perkin Elmer, 549 Albany Street, Boston, MA 02451, USA
}

Correspondence should be addressed to Bohumil Bednar, bohumil_bednar@merck.com

Received 23 April 2012; Accepted 6 September 2012

Academic Editor: Darrell R. Fisher

Copyright () 2012 Shu-An Lin et al. This is an open access article distributed under the Creative Commons Attribution License, which permits unrestricted use, distribution, and reproduction in any medium, provided the original work is properly cited.

\begin{abstract}
Inflammation as a core pathological event of atherosclerotic lesions is associated with the secretion of cathepsin proteases and the expression of $\alpha_{v} \beta_{3}$ integrin. We employed fluorescence molecular tomographic (FMT) noninvasive imaging of these molecular activities using cathepsin sensing (ProSense, CatB FAST) and $\alpha_{v} \beta_{3}$ integrin (IntegriSense) near-infrared fluorescence (NIRF) agents. A statistically significant increase in the ProSense and IntegriSense signal was observed within the chest region of apoE ${ }^{-/-}$ mice $(P<0.05)$ versus C57BL/6 mice starting 25 and 22 weeks on high cholesterol diet, respectively. In a treatment study using ezetimibe $(7 \mathrm{mg} / \mathrm{kg})$, there was a statistically significant reduction in the ProSense and CatB FAST chest signal of treated $(P<0.05)$ versus untreated $\mathrm{apoE}^{-/-}$mice at 31 and 21 weeks on high cholesterol diet, respectively. The signal of ProSense and CatB FAST correlated with macrophage counts and was found associated with inflammatory cells by fluorescence microscopy and flow cytometry of cells dissociated from aortas. This report demonstrates that cathepsin and $\alpha_{v} \beta_{3}$ integrin NIRF agents can be used as molecular imaging biomarkers for longitudinal detection of atherosclerosis, and cathepsin agents can monitor anti-inflammatory effects of ezetimibe with applications in preclinical testing of therapeutics and potentially for early diagnosis of atherosclerosis in patients.
\end{abstract}

\section{Introduction}

Atherosclerosis, a progressive inflammatory disease, results in the development of plaques within the arterial walls that may contain intracellular and extracellular lipids, a variety of inflammatory cells, extracellular matrix, smooth muscle cells, fibroblasts, and calcification [1-4]. Acute coronary syndrome (ACS) and stroke are typically associated with the development of vulnerable plaques. A vulnerable plaque is characterized by an accumulation of macrophages and macrophage-derived foam cells, degradation of the extracellular matrix, and the formation of a necrotic core, neoangiogenesis, intraplaque hemorrhage, and a thin fibrous cap [3, 5-7]. Thus, the prevention of a heart attack or stroke would benefit from the early detection of the biological processes resulting in the inflammation and neoangiogenesis associated with atherosclerotic lesions. The noninvasive detection of atherosclerotic plaque has been clinically limited mostly to the measurements of arterial stenosis in selected arterial beds [8-11]. Such measurements have been shown to be poor predictors of clinical outcomes, thus stimulating further research into the biology of atherosclerotic lesions and development of new biomarkers with a better predictive value for the formation of vulnerable plaques [12-17].

Inflammation of arterial walls is initially triggered by the accumulation of low-density lipoprotein (LDL) particles 
in the vessel wall and their subsequent oxidative and enzymatic modification followed by the activation of adhesion molecules and recruitment of T lymphocytes and monocytes [3]. Activated monocytes differentiate into macrophages, which accumulate modified LDL particles, evolving into foam cells $[18,19]$. As atherosclerosis progresses, endothelial cells, macrophages, and foam cells die, releasing matrix metalloproteinases and other intracellular enzymes such as cathepsins [20-22]. The released proteases cleave the extracellular matrix destabilizing the plaque core and the fibrous cap $[23,24]$. Transformation of monocytes into macrophages and eventually into foam cells is controlled by expression of extracellular receptors including $\alpha_{v} \beta_{3}$ integrin [19]. Another destabilizing factor of atherosclerotic lesions is angiogenesis, which is associated with $\alpha_{v} \beta_{3}$ integrin expression, and it is involved in cell-cell and cell-matrix adhesion [25]. The formation of new vessels originates from adventitial vasa vasorum and results in immature, fragile, and leaky vasculature, triggering intraplaque hemorrhage [26].

Molecular imaging biomarkers detecting metalloproteinase and cathepsin protease activity have been used by radiolabel-based [28] and optical imaging modalities [29] for the detection of inflammation in animal models of atherosclerosis and tissues from human atherosclerotic lesions [9, 30]. Cathepsin activatable, optical imaging agents based on cleavage of L-lysine sequences have been successfully applied in the characterization of atherosclerotic lesions in mouse and rabbit models of atherosclerosis and in the detection of cathepsin protease activity in human atherosclerotic plaques obtained at carotid endarterectomy [31-36]. Using these agents, fluorescence molecular tomography (FMT) and computed tomography (CT) have been applied to quantitatively evaluate the levels of inflammation in the mouse apoE $\mathrm{E}^{-/-}$ models of atherosclerosis [37]. Furthermore, agents with $\alpha_{v} \beta_{3}$ integrin targeted ligand have been employed to detect inflammation as well as angiogenesis in atherosclerotic lesions of animal models using a broad spectrum of imaging modalities [38-43].

Here we describe the development and evaluation of new cathepsin activatable agents and compare them to the new $\alpha_{v} \beta_{3}$ integrin targeting agent in a longitudinal study of atherosclerotic apo: $\mathrm{E}^{-/-}$mice undergoing prophylactic treatment with the anti-inflammatory therapeutic ezetimibe [44, 45] using FMT.

\section{Methods}

2.1. Determination of $I C_{50}$ in Cell Binding Assays. HEK293- $\alpha_{v} \beta_{3}$ cells were lifted with trypsin-EDTA and washed four times with serum-free MEM. Cells (25,000 cells/well) were added to microtiter wells coated with vitronectin or fibronectin and allowed to attach for 2 hours at $37^{\circ} \mathrm{C}$ in a humidified incubator in the absence or presence of increasing concentrations of IntegriSense or parent compound. Nonattached cells were gently washed away. Similar experiments were carried out with HEK293 cells stably transfected with $\alpha_{v} \beta_{5}, \alpha_{\mathrm{IIb}} \beta_{3}$, or $\alpha_{5} \beta_{1}$. The attached cells were quantified by colorimetric detection of hexosaminidase enzymatic activity in a Vmax plate reader (Molecular Devices, Menlo Park, CA). The number of attached cells was quantified using a standard curve for each cell line assayed and expressed as a mean value of triplicate samples.

2.2. Murine Atherosclerosis Model and Diet. Ten-week old apoE $^{-/-}$female mice $(n=184$ total: 20 for in vivo-ex vivo signal correlation study, 44 for longitudinal study, 120 for treatment study; Taconic Farms, Inc, Hudson, NY) fed on a $0.15 \%$ [wt/wt] cholesterol diet which included $9 \%$ of their caloric intake from fat (Research Diets, Inc, New Brunswick, NJ) were used as an animal model of atherosclerosis. In the longitudinal study, C57BL/6 mice ( $n=14$ total; Taconic Farms, Inc, Hudson, NY) fed on a normal chow diet served as controls. In the treatment study, apoE ${ }^{-/-}$mice were fed the diet described above with or without cholesterol absorption inhibitor ezetimibe $(7 \mathrm{mg} / \mathrm{kg} / \mathrm{d})$. All experiments were approved by our Institutional Animal Care and Use Committee (IACUC).

2.3. Correlation between In Vivo FMT Signal and Ex Vivo Fluorescence. Aged apoE ${ }^{-/-}$mice (8 to 10 months on diet), which developed atherosclerotic lesions, were injected with ProSense 750 ( $5 \mathrm{nmol}$ per animal), ProSense FAST 750 ( $8 \mathrm{nmol}$ per animal), or IntegriSense 750 (4 nmol per animal, all from Perkin Elmer, Boston, MA) via tail vein $24 \mathrm{hr}$ or $48 \mathrm{hr}$ before FMT imaging, respectively. Images were acquired by FMT2500 (Perkin Elmer) at $750 \mathrm{~nm}$ wavelength. The resulting $3 \mathrm{D}$ dataset was reconstructed and the region of interest (ROI) was defined within the chest area (Figure 2) using the vendor's software (TrueQuant, Perkin Elmer), and the threshold was set to $30 \%$ of the max concentration within the ROI. After in vivo imaging, animals were euthanized with $\mathrm{CO}_{2}$, and their aortas were excised. Fluorescence reflectance images of the dissected arteries (carotid arteries with aortic arch and thoracic part of descending aorta) were then acquired with the FMT2500.

2.4. FMT-CT Coregistration. To verify the in vivo FMT signal noninvasively, the mice were imaged with a GE Explore Locus Ultra (micro CT, GE) after FMT acquisition using a CT bed designed to hold the FMT animal cassette therefore ensuring the animal remained in the same position. The FMT dataset was exported in Dicom format and then coregistered to the CT dataset using Amira 5.2 (Visage Imaging, San Diego, CA).

2.5. Flow Cytometry. After probe-injected mice were euthanized, their aortas were excised and terminal blood samples were collected. The aortas were minced with fine scissors and dissociated into single cells by incubating with Liberase $\mathrm{TH}$ and TM (Roche Applied Science, Indianapolis, IN) at $37^{\circ} \mathrm{C}$ for 1.5 hours. The resulting cell suspensions were then passed through $40 \mu \mathrm{m}$ cell strainer (BD Biosciences, Bedford, MA) and washed with PBS. The blood samples were incubated in ACK buffer (Cambrex, Walkersville, MD) to remove blood cells and then washed with PBS. For multicolor flow cytometry analysis of lymphoid cells, myeloid cells (including 
monocytes/macrophages and neutrophils) and other cells (including stromal cells of the aortic wall), cells were incubated with a cocktail of mAbs (BD Biosciences, San Francisco, CA) against T cells (CD90-FITC), B cells (B220FITC), NK cells (CD49b-FITC, NK1.1-FITC), and myeloid cells (CD11b-PE) at $4^{\circ} \mathrm{C}$ for $30 \mathrm{~min}$ and then analyzed on an LSRII (BD Biosciences, San Francisco, CA). Acquisition data was analyzed by the FlowJo software (Tree Star Inc., Ashland, OR).

2.6. Immunofluorescence Microscopy. To demonstrate colocalization of macrophages and the IntegriSense 680 and ProSense 680 probes, selected vessels were stained with a monoclonal anti-CD68 antibody. Ten micron-thick cryosections were prepared and fixed in ice-cold acetone/ethanol $(3: 1)$ for $10 \mathrm{~min}$. and washed in PBS. Nonspecific binding was blocked by incubation with $5.0 \%$ nonimmune goat serum for $30 \mathrm{~min}$ followed by incubation with the rat antimouse CD68 antibody ( $5 \mu \mathrm{g} / \mathrm{mL}$; MCA-1957, AbD Serotec, Raleigh, NC) for $1 \mathrm{hr}$ at room temperature. Sections were washed in PBS and incubated with AlexaFluor 488 goat antirat IgG $(4 \mu \mathrm{g} / \mathrm{mL}$; Molecular Probes, Eugene, OR) for $1 \mathrm{hr}$ and again washed in PBS. Sections were mounted using a fluorescent mount medium with or without DAPI counterstain and examined with a Nikon E-1000 microscope equipped with the appropriate band-pass filters.

2.7. Longitudinal Study Design. For the longitudinal study, both $\mathrm{ApoE}^{-/-}(n=15$ for each agent) and C57BL/6 $(n=9$ for ProSense, $n=5$ for IntegriSense) mice were injected with ProSense750 or IntegriSense750 and imaged with the FMT2500 as described above. The scans were conducted at the times indicated in the figures.

For the treatment study, ProSense, CatB FAST agent or ProSense FAST ( $8 \mathrm{nmol}$ per animal, Perkin Elmer) were injected into apoE ${ }^{-/-}$mice (both treated; $n=15$ for each agent, and untreated; $n=15$ for each agent) via tail vein $24 \mathrm{hr}$ before imaging. IntegriSense $(2 \mathrm{nmol}$ per animal, Perkin Elmer) injected mice were images $48 \mathrm{hr}$ after injection. The imaging acquisition was carried out at the time points as depicted in Figures 7 and 8.

2.8. Quantitative Immunohistochemical Analysis. After apoE ${ }^{-1-}$ mice were euthanized, their aortas were excised and fixed in 10\% buffered formalin (Figure 6(a)). The aortas were then divided into six levels as indicated in Figure 6(b). All vessels were embedded into paraffin blocks, sectioned at $4 \mu \mathrm{m}$, and placed onto adhesive-coated slides. Six slides per level were taken at $\sim 50 \mu \mathrm{m}$ intervals. Slides were stained with hematoxylin and eosin to demonstrate general morphology and immunostained with $\mathrm{mAbs}$ against the F4/80 antigen ( $1: 100$, eBioscience, Inc., San Diego, CA). Slides were subsequently incubated with a peroxidase-conjugated anti-mouse IgG antibody and 3' 3 '-diaminobenzidine (DAB) chromogen to identify macrophages within the plaque regions and counterstained with hematoxylin (Figure 6(c)). The stained slides were analyzed using the Ariol image analysis platform (Genetix, San Jose, CA) for macrophage quantification.
Regions of interest were drawn to include only the plaque area and appropriate size, and color classifiers were applied to specifically identify the DAB stained (brown) macrophages, which were then counted.

2.9. Serum Cholesterol Concentration. Terminal serum samples $(n=12)$ were collected at the end of the treatment study, and the HDL-C and total cholesterol levels were determined by following the manufacturer's protocol of HDLCholesterol E kit (Wako Chemicals, Richmond, VA) with and without adding precipitating reagent, respectively.

2.10. Plasma Ezetimibe Concentration. Terminal plasma samples $(n=12)$ were collected at the end of the treatment study and assayed for and ezetimibe-glucuronide using Aria LC-MS/MS with a standard calibration range from 5 to $10,000 \mathrm{nM}$ and a quantification limit of $5 \mathrm{nM}$.

2.11. Statistics. Data are presented as mean \pm SEM. For statistical analysis, we used ANOVA followed by Bonferroni $t$-test for multiple comparisons. Differences were considered significant at $P<0.05$. The correlation between the in vivo and ex vivo signal was evaluated using linear regression analysis.

\section{Results}

3.1. Molecular and Imaging Characteristics of Cathepsin and $\alpha_{v} \beta_{3}$ Integrin Agents. Structural schematics, spectral characteristics, and the activation selectivity of the cathepsin agents and a chemical structure of $\alpha_{v} \beta_{3}$ integrin agent used in our experiments are depicted in Figure 1. The cathepsin activated agent ProSense (Figures 1(a) and 1(b)), used in the detection of atherosclerotic lesions before [34-37], is based on $\sim 400 \mathrm{kDa}$ polylysine macromolecules with covalently attached fluorophores [27]. The newly developed cathepsin agents ProSense FAST and Cat B FAST are based on low molecular weight substrate peptides with fluorochromes attached to the $\mathrm{C}$ - and $\mathrm{N}$-termini of the peptide resulting in $95 \%$ of the fluorescence quenching and a hypsochromic ("blue") shift of about $60 \mathrm{~nm}$ in the absorption spectra (Figures 1(c) and 1(d)). The quenching of the fluorophores in the ProSense agent is achieved by tight packing of the polymer coil, which slows the activation of this agent. Thus, the full activation of the ProSense agent requires 24 hours while the newly designed peptide-based agents are fully activated within 6 to 8 hours (data no shown). The selectivity of the agents for activation by 24 hours incubation with different cathepsins is shown in Figure 1(e). Both ProSense and ProSense FAST agents were activated by most of the evaluated cathepsins, while the Cat B FAST agent was activated mostly by cathepsin B (Figure 1(e)).

The $\alpha_{v} \beta_{3}$ targeted agent, IntegriSense, is based on a low molecular weight peptidomimetic ligand of $\alpha_{v} \beta_{3}$ integrin, which was chemically modified to covalently attach the VivoTag (NIRF) (Figure 1(f)). The affinity of the agent for activated $\alpha_{v} \beta_{3}$ integrin was determined to be $\sim 0.5 \mathrm{nM}[46]$ and the agent is highly selective for $\alpha_{v} \beta_{3}$ (Table 1 ). 


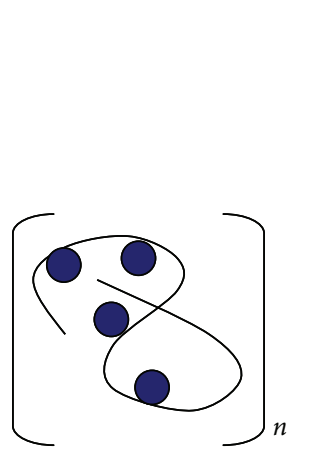

(a)

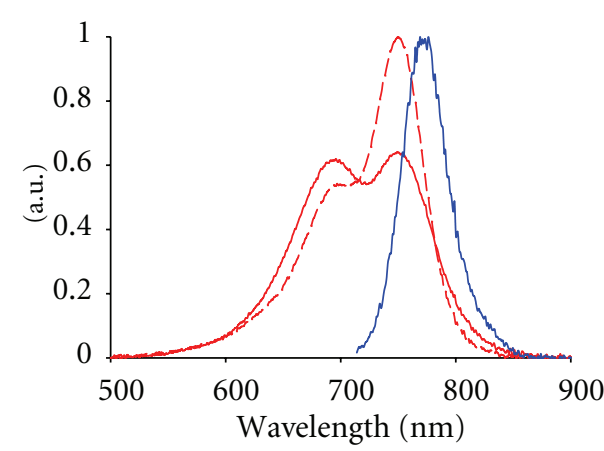

(b)

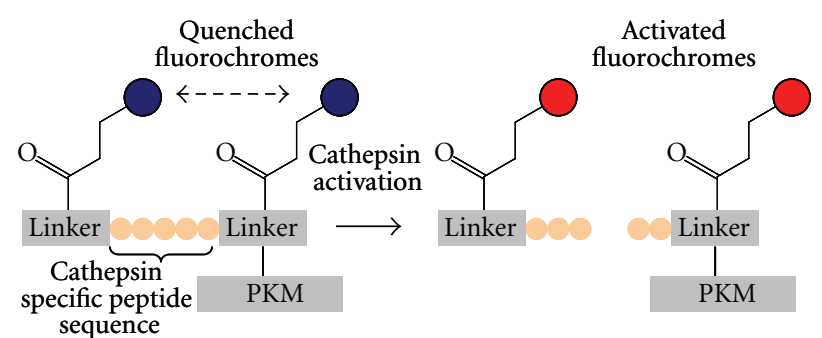

(c)
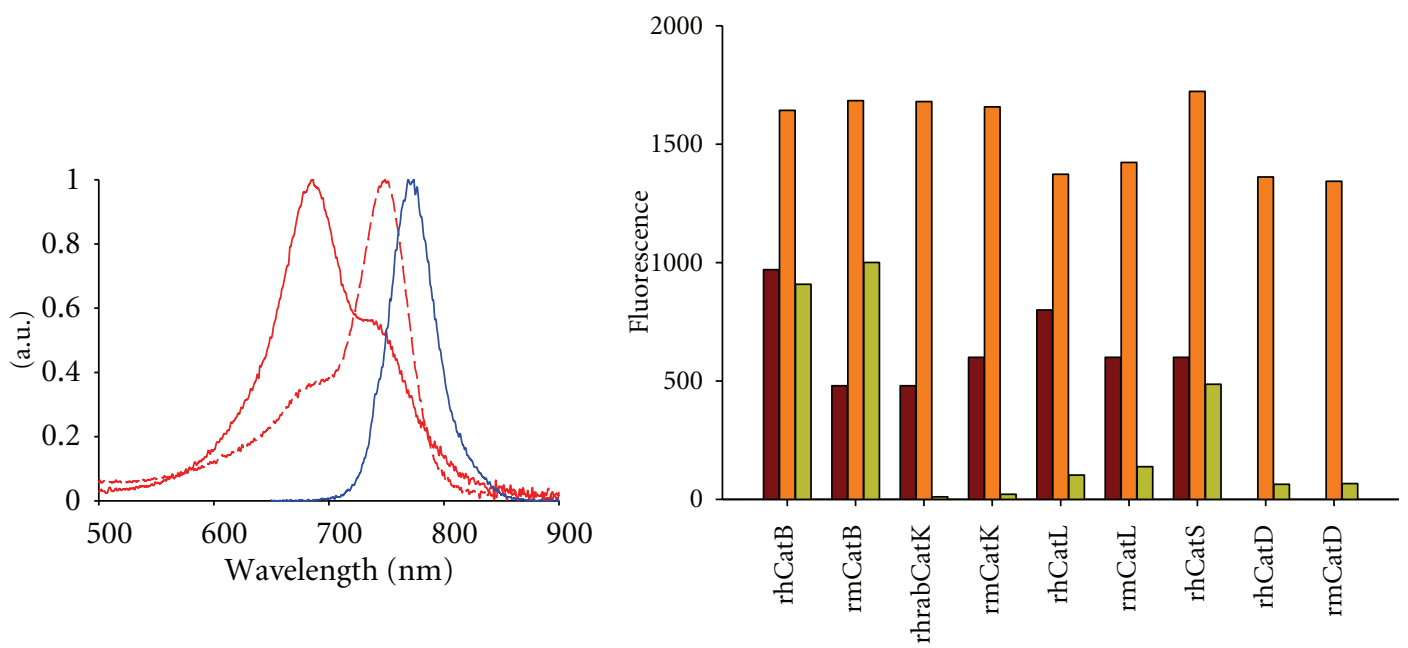

ProSense 750
ProSense FAST 750

CatB FAST

(d)

(e)<smiles>Cc1ncc([C@H](CCCCCCc2ccc3c(n2)NCCC3)CC(=O)O)cn1</smiles>

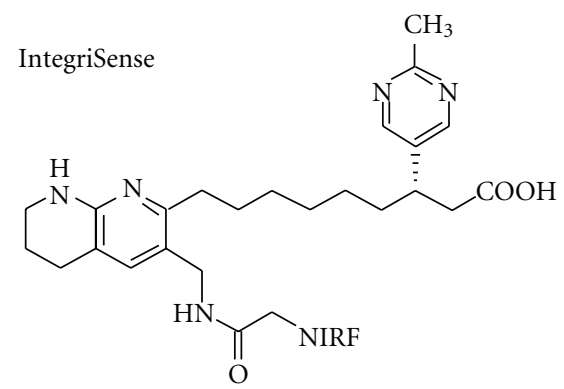

(f)

FIGURE 1: Basic characteristics of cathepsin and $\alpha_{v} \beta_{3}$ integrin agents. (a), (b) Cartoon and spectra of ProSense $\sim 400 \mathrm{kDa}$ polylysinebased [27]; (c), (d) Cartoon and spectra of FAST peptide-based agents $\sim 23 \mathrm{kDa}$, PKM: pharmacokinetic modifier, absorption spectra (before activation-dash red and after activation: solid red), emission spectra: solid blue; (e), selectivity in cathepsin probe activation at 24 hours incubation; (f), Chemical structure of parent $\alpha_{v} \beta_{3}$ integrin ligand (top) and IntegriSense (bottom, 1278 Da) NIRF: VivoTag fluorophore. 


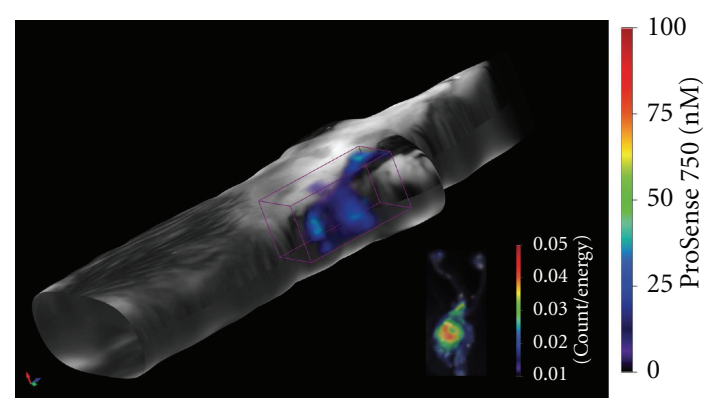

(a)

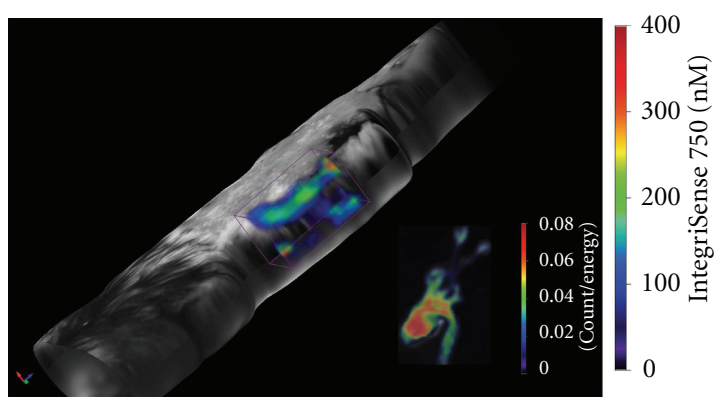

(c)

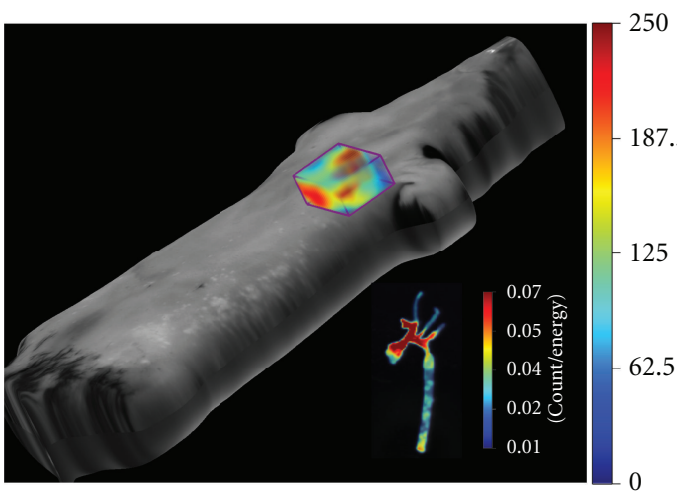

(e)

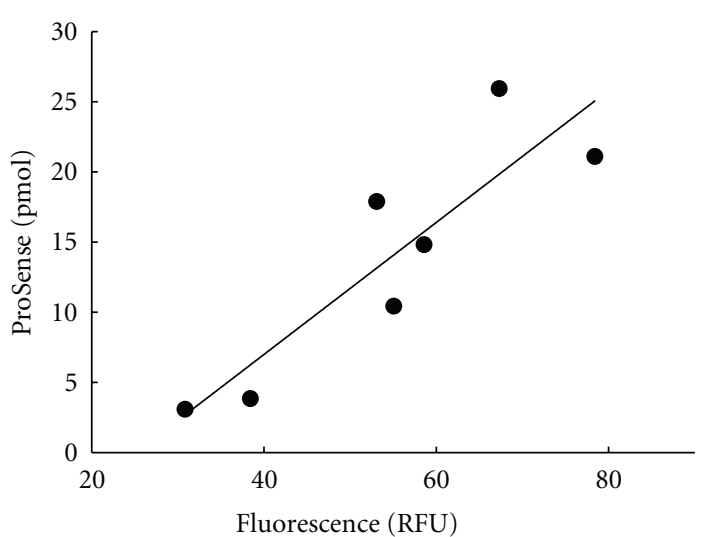

(b)

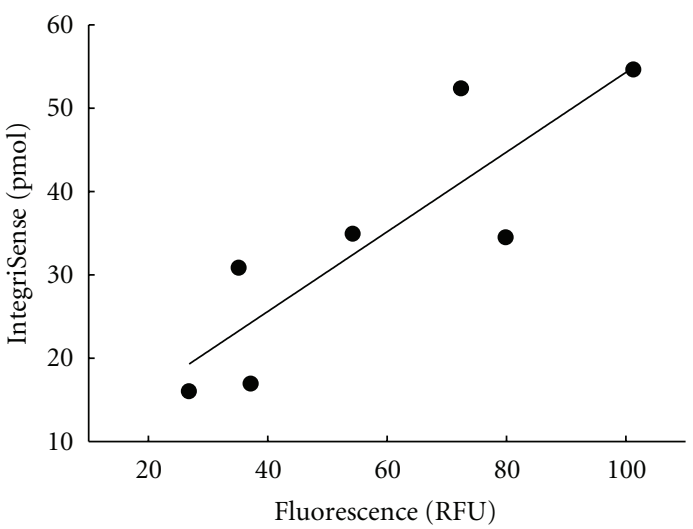

(d)

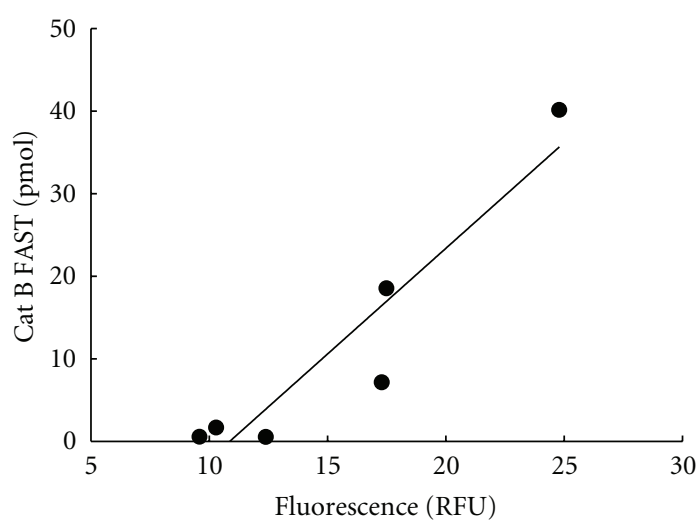

(f)

Figure 2: Examples of in vivo FMT images of $\mathrm{apoE}^{-/-}$mice and ex vivo fluorescence images of the corresponding areas of dissected arteries and correlation between the in vivo and ex vivo measurements. (a), (b) ProSense $(r=0.89, P<0.05)$; (c), (d) IntegriSense $(r=0.86$, $P<0.05)$. (e), (f) Cat B FAST $(r=0.94, P<0.05)$. Plots for ProSense and IntegriSense are based on seven and for Cat B FAST on six different animals.

3.2. Agents Detected In Vivo Using FMT Correlate with Ex Vivo Fluorescence Measurements of Dissected Arteries. To assess the severity of atherosclerotic disease, we focused on the aortic arch and the proximal portion of the descending aorta. Since there is limited anatomical information to guide in vivo FMT analysis, we compared fluorescent signal within the ROI drawn to capture the entire volume of the chest region with the signal measured within the ROI drawn on the excised arteries (for details see Methods) to ensure the accuracy of the in vivo quantification (Figures $2(\mathrm{a})-2(\mathrm{f}))$. The total amount of the probes (pmol) detected in vivo correlated well with the fluorescence (counts/energy) measured ex vivo for all evaluated agents (Figures 2(b), 2(d), and 2(f)). This suggests that the in vivo quantification reflects the actual 


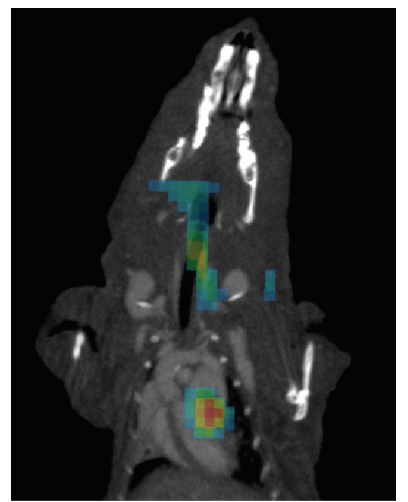

(a)

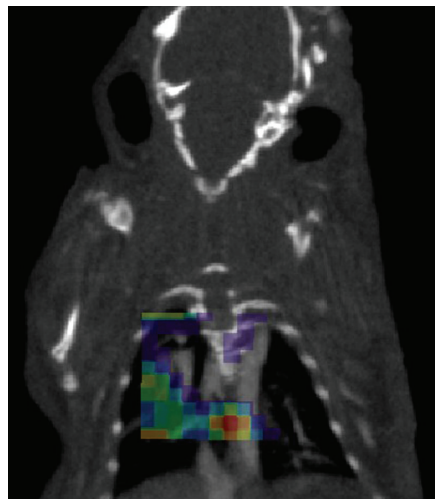

(b)

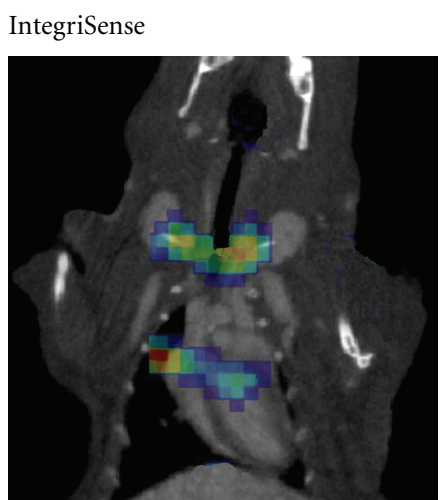

(c)

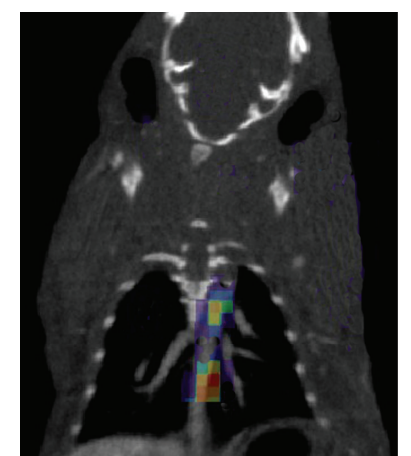

(d)

FIgURE 3: Coregistration of FMT fluorescence images with CT anatomical images. (a) (Aortic root and arch and carotid arteries), (b) (proximal part of descending aorta) using Cat B FAST, (c) (aortic root and arch and carotid arteries), (d) (proximal part of descending aorta) using IntegriSense.
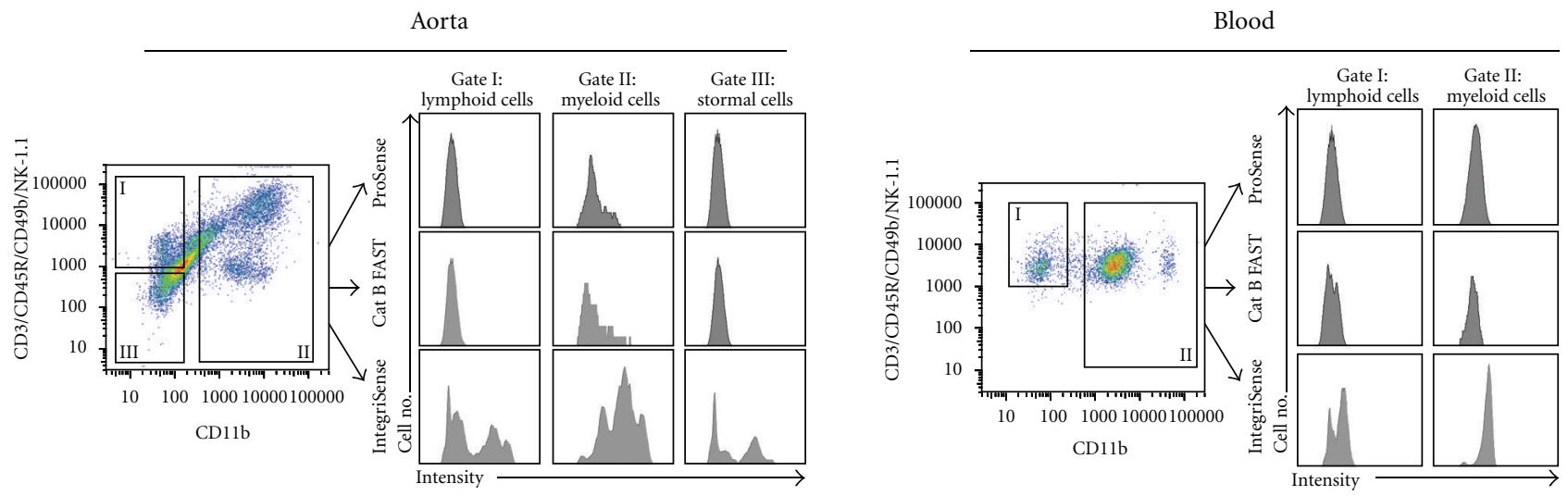

(a)
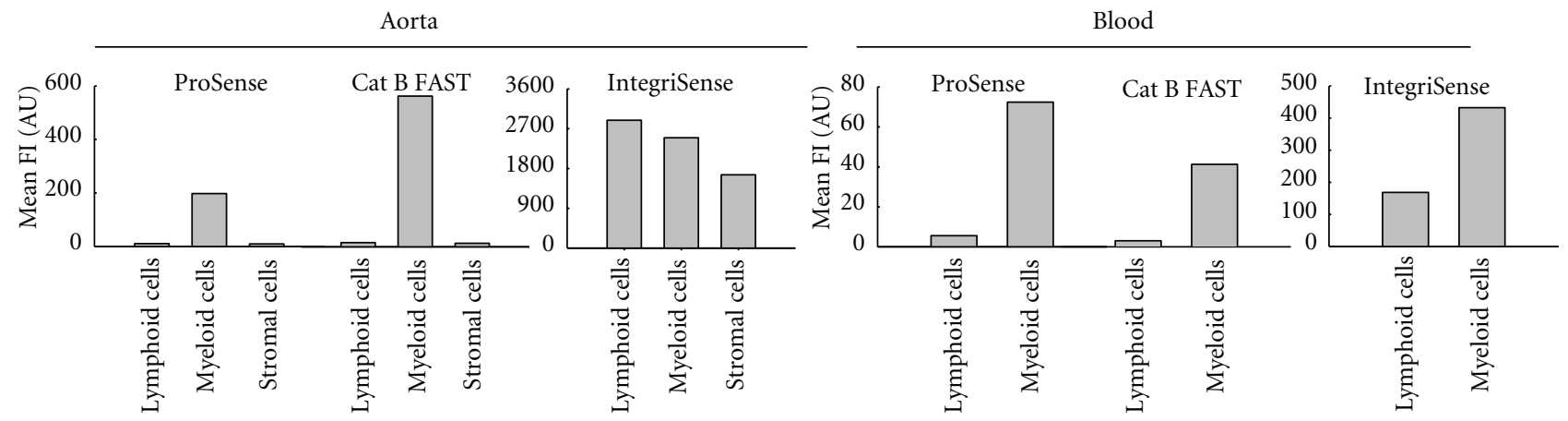

(b)

FIGURE 4: Flow cytometric analysis of the agent's fluorescence in the cells isolated from dissected arteries and blood of apoE $E^{-/-}$mice on high cholesterol diet 24 hours after injection of cathepsin and IntegriSense agents. (a) Cells were before flow cytometry incubated with an antibody cocktail, which allows for selective identification of lymphoid, myeloid cells including monocytes/macrophages and neutrophils and other cells such as stromal cells of the aortic wall. (b) Mean fluorescence histograms of these cells show the highest distribution of cathepsin probes in the myeloid cells, while the $\alpha_{v} \beta_{3}$ integrin agent was found in all types of identified cells. In the blood, the relative distribution of all agents is similar. However, the absolute signal of cathepsin probes is in blood much lower than in arterial cells, while the distribution of $\alpha_{v} \beta_{3}$ integrin agent is higher. 


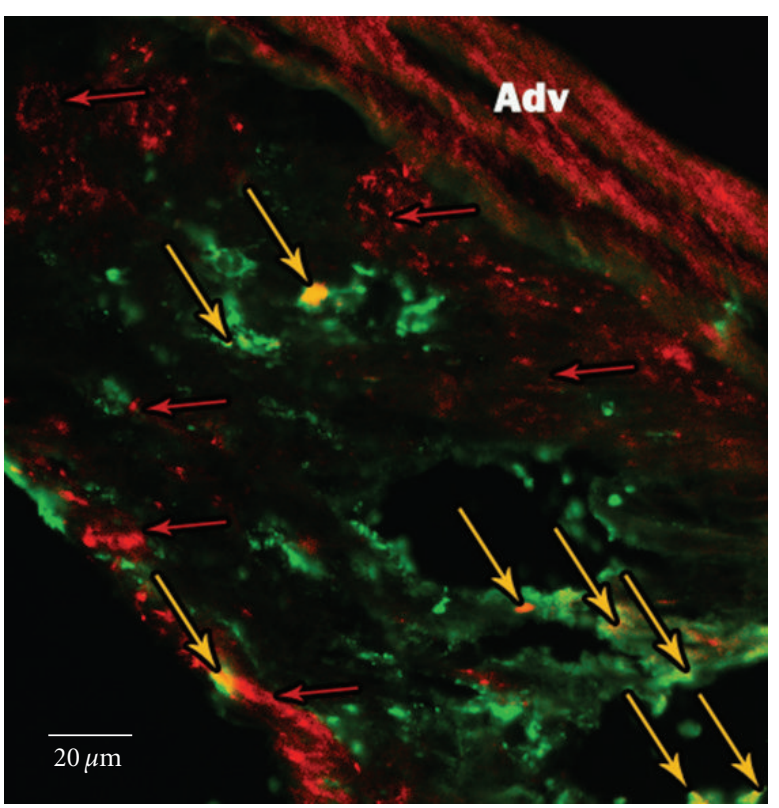

(a)

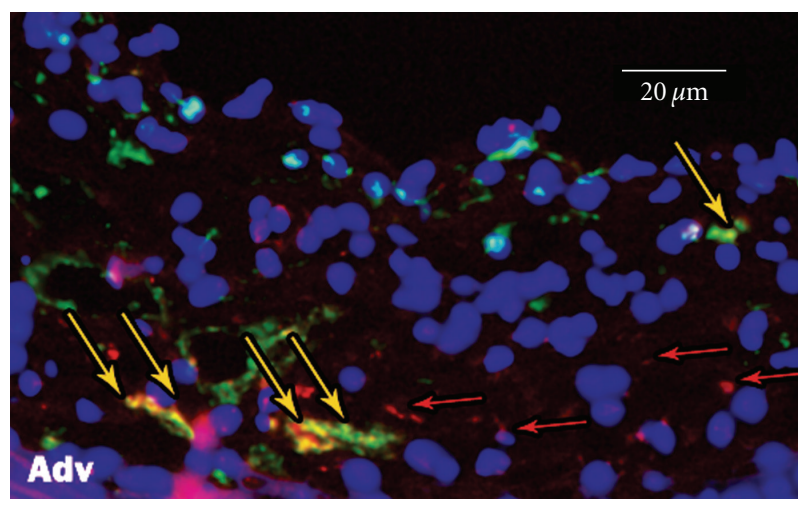

(b)

FIGURE 5: Immunofluorescence histochemistry of the sections from the aortic arch $a \mathrm{poE}^{-/-}$mice on a high cholesterol diet. (a) IntegriSense, (b) ProSense, red: imaging agent, green: CD 68 antibody staining monocytes/macrophages, blue: DAPI staining of cell nucleus, yellow arrows: colocalization of agents and macrophages, red arrows: distribution of agents in the core of atherosclerotic lesion, Adv: adventitia.

signal from the area of interest and can therefore be used to measure the inflammation non-invasively using fluorescence of these imaging agents.

\subsection{Coregistration of Fluorescence FMT Signal and an Anato-} mical CT Image. To further identify the location of the signal, we co-registered the FMT signal with CT images based on fiducial landmarks that are incorporated into the animal holder and are identified by both FMT and CT. Using anatomical detail from the CT image combined with FMT molecular information, we determined that the majority of the fluorescent signal was in the aortic root, arch, and the proximal portion of the descending aorta of animals injected
TABLE 1: Measurement of IntegriSense selectivity in binding to different integrin receptors $\mathrm{IC}_{50}$ values (mean \pm SEM) of IntegriSense 750 and Parent Compound in cell adhesion assays.

\begin{tabular}{lcc}
\hline & \multicolumn{2}{c}{$\mathrm{IC}_{50}(\mathrm{nM})$} \\
& IntegriSense 750 & Parent compound \\
\hline$\alpha_{\mathrm{v}} \beta_{3}$ & $3.5 \pm 0.2$ & $0.82 \pm 0.2$ \\
$\alpha_{\mathrm{v}} \beta_{5}$ & $41 \pm 12$ & $1.3 \pm 0.2$ \\
$\alpha_{\mathrm{IIb}} \beta_{3}$ & $48 \pm 15$ & $>10,000$ \\
$\alpha_{5} \beta_{1}{ }^{*}$ & $>5,000$ & $>10,000$ \\
\hline
\end{tabular}

${ }^{*}$ Binding assays to vitronectin were performed for all integrin receptors except $\alpha_{5} \beta_{1}$ which was performed on fibronectin.

with cathepsin-activated (Figures 3(a) and 3(b) or $\alpha_{v} \beta_{3}$ targeted agents (Figures 3(c) and 3(d)).

3.4. Cellular Target Profile by Flow Cytometry. To validate the cellular source of the fluorescence signal in vivo, we performed flow cytometry of single cell suspensions generated from digested murine aortas. Monocytes/macrophages were identified as the dominant cellular signal source for all cathepsin activated agents tested. We observed a similar labeling profile in the blood; however, the signal intensity was 10 times lower compared to the aortic signal. Fluorescence signal from the $\alpha_{v} \beta_{3}$ targeted agent, IntegriSense, was found not only in monocytes/macrophages but also in lymphoid and stromal cells. The signal from IntegriSense was higher in monocytes/macrophages than in lymphoid cells in the blood (Figure 4).

3.5. Immunofluorescence Microscopy Reveals Colocalization of Fluorescence of Optical Agents with Macrophages. The microscopic fluorescence distribution of both cathepsin activated and $\alpha_{v} \beta_{3}$ targeted agents was compared to immunoreactive staining for macrophages CD68 (Figure 5). There was good colocalization of fluorescence from the optical agents with immunofluorescent CD68 signal, while some of $\alpha_{v} \beta_{3}$ agent was found located in the adventitia.

3.6. In Vivo FMT Quantification versus Ex Vivo Macrophage Count. Macrophages have been shown to play an important role in the inflammatory process of atherosclerosis $[1,2$, $5,18]$. To evaluate the prognostic value of different optical agents for non-invasive detection of the disease, quantitative immunohistochemical analysis of macrophages in aortic arteries using F4/80 monoclonal antibodies was performed, and the macrophage counts were then correlated with in vivo FMT measurements of the same animals. Quantification of the in vivo FMT signal and ex vivo macrophage count showed a good correlation for both ProSense $(r=0.89, P<0.05)$ and Cat B FAST $(r=.86, P<0.05)$, but not for IntegriSense $(r=$ $0.35, P=0.44$ ) (Figures 6(d)-6(f)).

3.7. Longitudinal Imaging Using Cathepsin B Sensing and $\alpha_{v} \beta_{3}$ Integrin Optical Agents in apoE ${ }^{-/-}$Mice. To access cathepsin activity or $\alpha_{v} \beta_{3}$ integrin expression as a function of time throughout disease progression, we performed in vivo FMT 


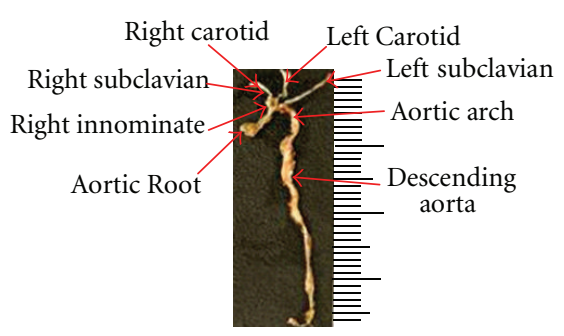

(a)

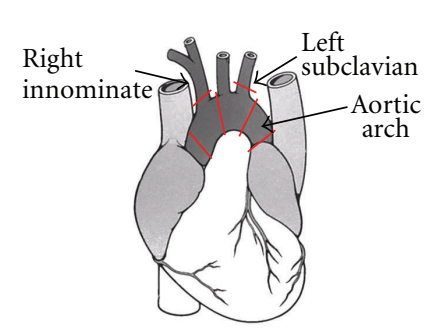

(b)

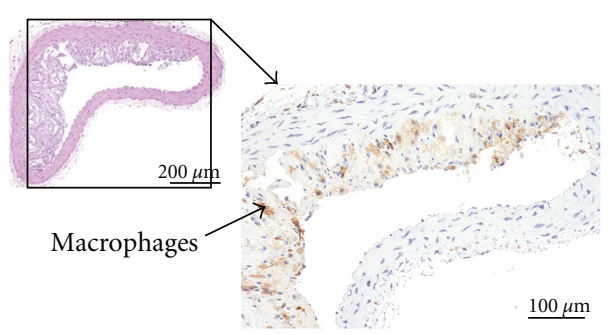

(c)

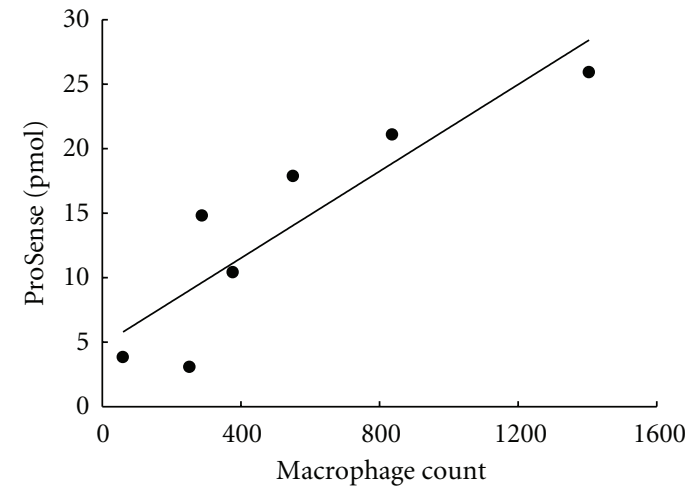

(d)

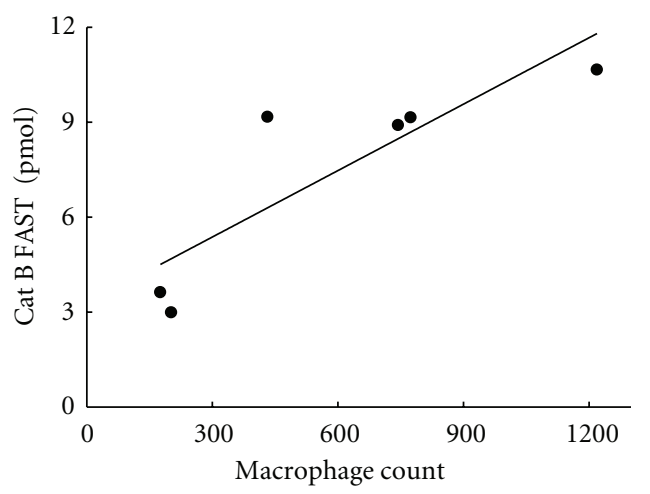

(e)

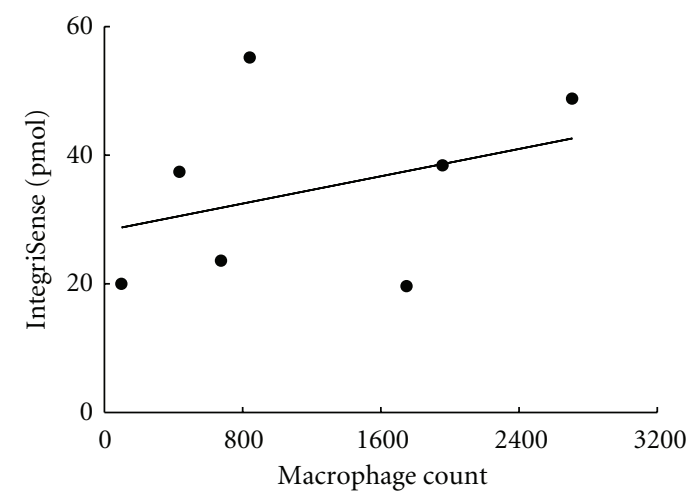

(f)

FIGURE 6: Correlation between quantitative immunohistochemical analysis of the macrophage content and the FMT measured agent amount in selected sections from apoE ${ }^{-/-}$mice on high cholesterol diet. (a) An example of dissected arteries from apoE ${ }^{-/-}$mice. (b) A mouse heart cartoon with six regions marked with red lines, where the arteries were sectioned for histology. (c) An example of stained section used in the macrophage quantification. (d), (e), (f) Plots of total agent amount measured in vivo by FMT as a function of macrophage count for ProSense ((d), $r=0.89, P<0.05, n=7)$, Cat B FAST ((e), $r=0.86, P<0.05, n=6)$, and IntegriSense $((\mathrm{f}), r=0.35, P=0.44, n=7)$, respectively.

imaging in both apoE ${ }^{-/-}$and $\mathrm{C} 57 \mathrm{BL} / 6$ mice at time points indicated in Figure 7. A statistically significant increase in the amount of ProSense and IntegriSense was observed in the chest area of apoE ${ }^{-/-}$versus C57BL/6 mice starting 25 and 22 weeks on diet, respectively.

3.8. Cathepsin Agents but Not $\alpha_{v} \beta_{3}$ Integrin Agent Detect Inhibition of Inflammation by Ezetimibe. We further evaluated the newly developed agents for the detection of reduced inflammation associated with atherosclerotic lesions in apoE $E^{-1-}$ mice upon prophylactic treatment with the cholesterol lowering agent, ezetimibe. Ezetimibe is known to lower
LDL and inflammation associated with the atherosclerosis in apo: $\mathrm{E}^{-/-}$mice [44]. In our experiments, ezetimibe lowered the LDL and elevated HDL levels about threefold (Table 2). Cat B FAST detected a statistically significant effect of the treatment at both 21 and 31 weeks on the diet (Figure 8(c)), while the large, nonselective, polymer-based agent, ProSense, identified a statistically selective effect of the treatment only at 31 weeks on diet (Figure 8(a)). The newly developed nonselective agent, ProSense FAST, found a statistically significant effect of the treatment only at 12 weeks (Figure 8(b)) while IntegriSense was insensitive to the effect of treatment by ezetimibe (Figure $8(\mathrm{~d})$ ). 


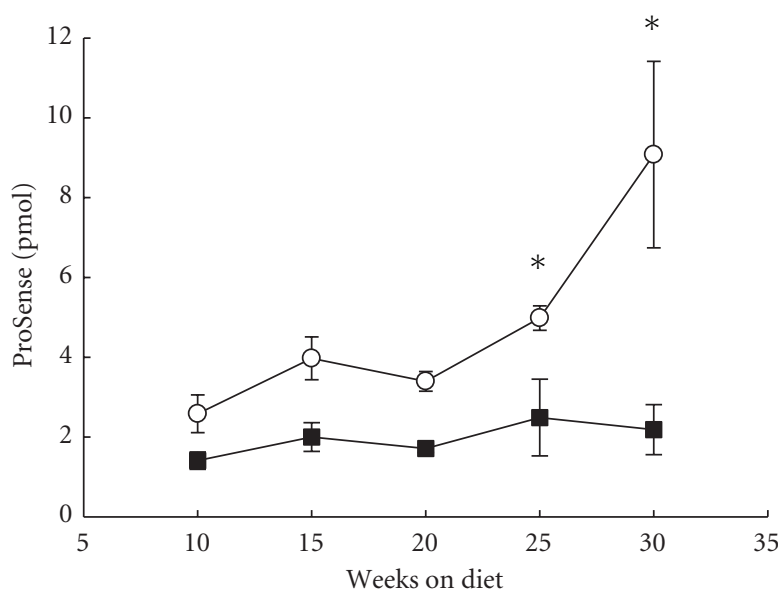

$\rightarrow-\mathrm{C} 57 \mathrm{BL} / 6$

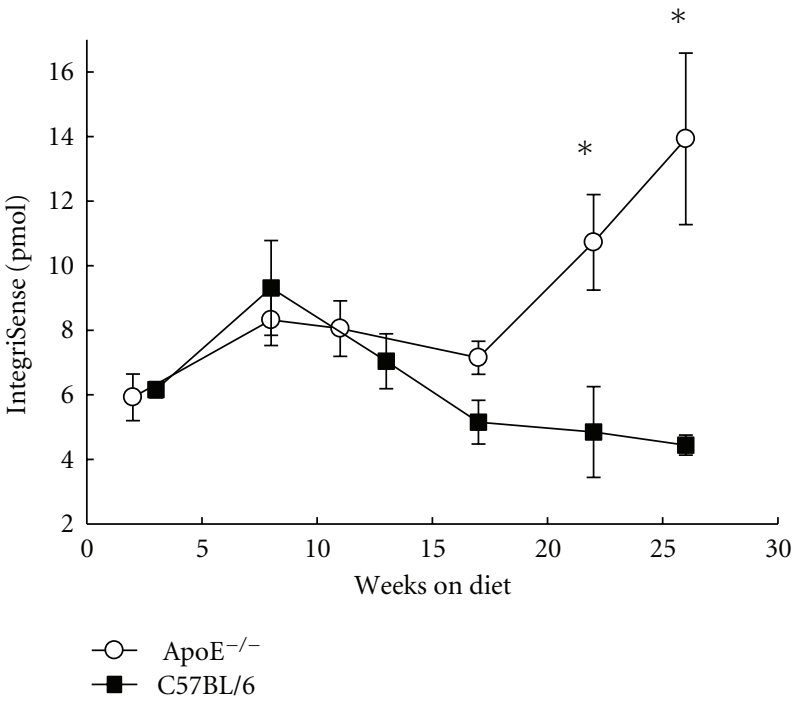

(b)

Figure 7: Quantitative in vivo FMT measurements of agents in the thorax area of apoE ${ }^{-/-}$mice $(n=15$ in each group, total 30 animals) on a high cholesterol and control C57BL/6 mice $(n=9$ for ProSense, $n=5$ for IntegriSense study) on regular chow diet. (a) ProSense; (b) IntegriSense (mean \pm SEM, $\left.{ }^{*} P<0.05\right)$.

TABLe 2: Serum cholesterol levels and plasma ezetimibe concentration $\left(\right.$ Mean \pm SD) of ezetimibe treated and control apoE $E^{-/-}$mice.

\begin{tabular}{lccc}
\hline Group & $\begin{array}{c}\text { HDL-C } \\
(\mathrm{mg} / \mathrm{dL})\end{array}$ & $\begin{array}{c}(\mathrm{LDL}+\mathrm{VLDL})-\mathrm{C} \\
(\mathrm{mg} / \mathrm{dL})\end{array}$ & $\begin{array}{c}\text { Ezetimibe } \\
(\mu \mathrm{M})\end{array}$ \\
\hline Control & $11.5 \pm 0.9$ & $411 \pm 59$ & Undetectable \\
Treated & $32.5 \pm 2.9$ & $106 \pm 27$ & $0.55 \pm 0.11$ \\
\hline
\end{tabular}

\section{Discussion}

Heart attack and stroke, often fatal clinical outcomes of atherosclerosis, are frequently associated with vulnerable arterial plaques that develop as a product of inflammatory processes initiated by high levels of LDL [1]. Thus, molecular imaging biomarkers of inflammation may play an important role in the monitoring the development and efficacy of new therapeutics as well as in the early detection of the disease. In this paper, we describe the development and evaluation of new cathepsin activatable agents and their comparison to the new $\alpha_{v} \beta_{3}$ integrin targeted agent in the detection of atherosclerosis associated inflammation in $\mathrm{apoE}^{-/-}$mice using established FMT technology. We further demonstrate the sensitivity of individual biomarkers to detect inhibition of inflammation in prophylactic studies with the therapeutic ezetimibe.

We have developed new cathepsin agents, ProSense FAST and Cat B FAST, based on low molecular weight peptides flanked by fully quenched fluorophores. Once the protease cleaves the low molecular weight peptide, the fluorophores diffuse apart leading to a full fluorescence signal. These agents reach maximum activation within six to eight hours, while the polymer-based agent ProSense [27, 37] requires 24 hours for full activation. Activation of ProSense FAST is nonselective for tested cathepsins similar to the polymer-based ProSense, which is only weakly selective for cathepsin B. Conversely, the Cat B FAST agent is highly selective for cathepsin B. Thus, parallel measurements using the new agents and polymer-based ProSense can address the effect of the protease selectivity and to some extent also the potential effect of the polymer coil on the detection sensitivity of atherosclerosis associated inflammation.

The $\alpha_{v} \beta_{3}$ integrin targeted agent, IntegriSense, has been used successfully in the detection of tumor growth [46] and RGD-based $\alpha_{v} \beta_{3}$ integrin targeted agents in the detection of inflammation and angiogenesis in mouse and rabbit models of atherosclerosis [40-42]. We evaluated the sensitivity and specificity of IntegriSense in the detection of atherosclerotic lesions in the apoE $\mathrm{E}^{-/-}$mice model of atherosclerosis and compared it to the cathepsin-activatable agents. All new agents were developed with the requirements for the design of low molecular weight therapeutics. Fluorophores were selected to align with the parameters needed to stay within the optimal tissue transparency window (680-950 nm) [27, 30 ], with potential for future successful applications in the clinical evaluation of patients.

We employed FMT technology for the quantitative in vivo detection of molecular imaging biomarkers in both apoE $^{-/-}$and control C57BL/6 mice and confirmed the localization of the measured fluorescence using CT. We found that the FMT measured concentrations of imaging biomarkers in the thoracic area of apoE $\mathrm{E}^{-/-}$mice indicate that these agents accumulated in the aortic arch and the roots of adjacent carotid and subclavian arteries (sites of histologically confirmed atherosclerosis) similar to previous reports [37]. Moreover, the in vivo measured agent concentrations of both 


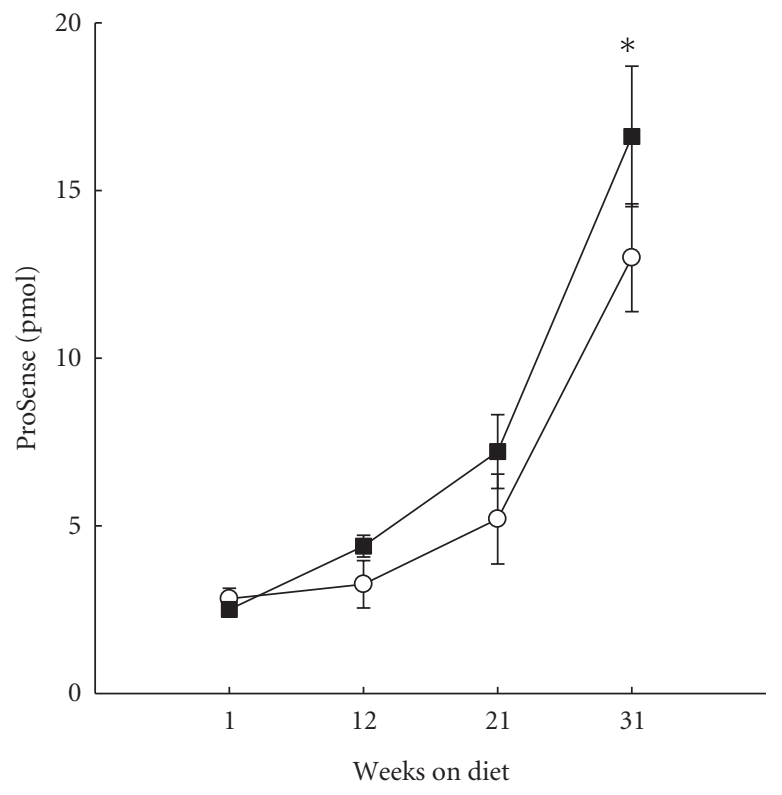

(a)

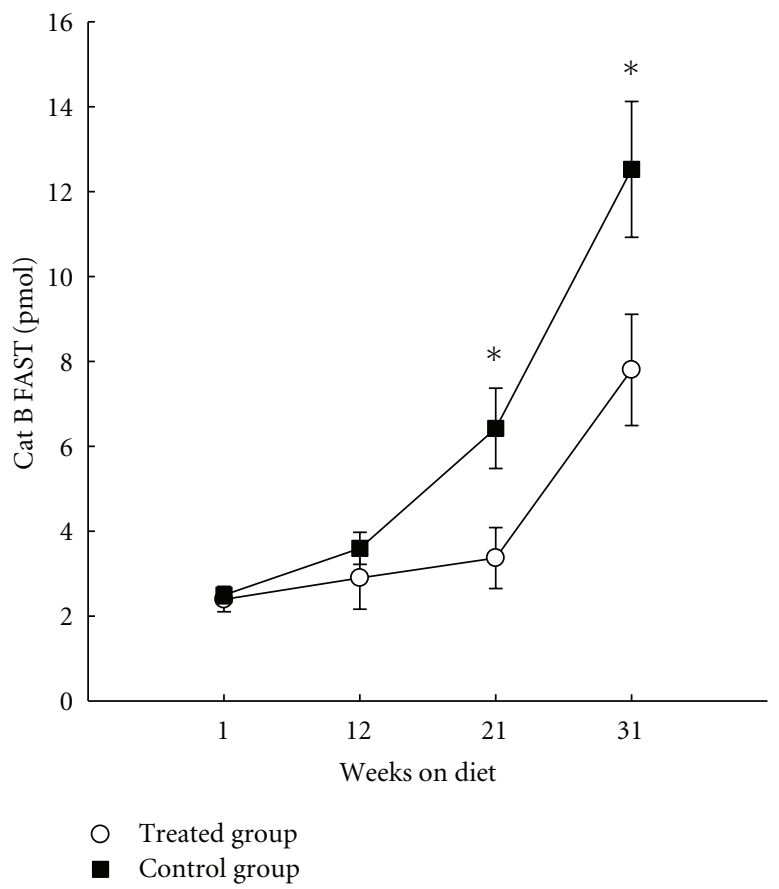

(c)

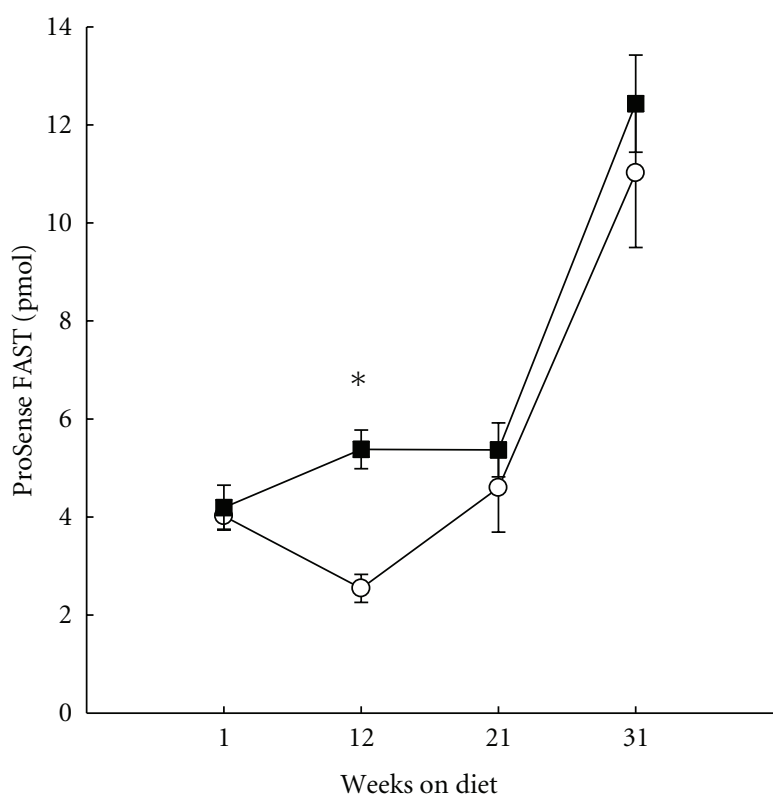

(b)

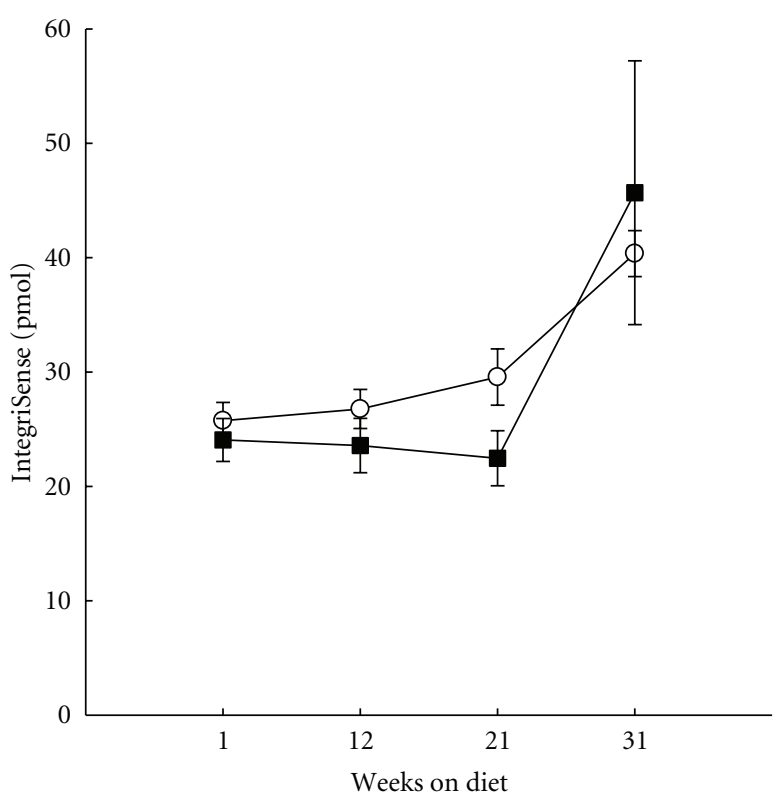

○ Treated group

- Control group

Figure 8: The effect of prophylactic treatment using ezetimibe on the FMT measured levels of agents in thorax areas of apoE ${ }^{-1-}$ mice $(n=15$ in each group, total 120) on high cholesterol diet. (a) ProSense; (b) ProSense FAST; (c) Cat B FAST; (d) IntegriSense (mean \pm SEM, $\left.{ }^{*} P<0.05\right)$.

cathepsin-activated and $\alpha_{v} \beta_{3}$ integrin targeted agents correlated well with the ex vivo fluorescence in the same areas of the dissected arteries of apoE $\mathrm{E}^{-/-}$mice.

An early stage of atherosclerotic lesions is characterized by infiltration of monocytes and macrophages and their development into foam cells [1-3] controlled by extracellular receptors including $\alpha_{v} \beta_{3}$ integrin [19]. As the atherosclerotic plaque progresses, endothelial cells, macrophages, and foam cells die, releasing matrix metalloproteinases and other intracellular enzymes such as cathepsins [20-22, 24]. The cell proliferation triggers on $\alpha_{v} \beta_{3}$ integrin-dependent angiogenesis, originating from adventitial vasa vasorum and resulting in immature, fragile, and leaky vasculature, triggering intraplaque hemorrhage $[25,26]$. To understand the cellular profile of the imaging biomarkers, we conducted flow cytometric analysis of cells isolated from dissected 
aortic tissues of apoE $\mathrm{E}^{-/-}$mice and showed that cathepsin agents were activated primarily in myeloid (monocytes and macrophages) cells while the $\alpha_{v} \beta_{3}$ integrin agent was found not only in myeloid cells but also in lymphoid and stromal (endothelial) cells indicating that IntegriSense likely detects inflammatory cells, angiogenesis, and cell proliferation. Flow cytometry of blood from animals injected with cathepsin agents detected very low levels (compared to aortas) of the agents in both myeloid and lymphoid cells 24 hours after agent injection, while $\alpha_{v} \beta_{3}$ agent was also detected in both cell types, albeit at much higher levels for myeloid cells, indicating again involvement of $\alpha_{v} \beta_{3}$ integrin in the development and proliferation of inflammatory cells.

Immunofluorescence detected the activated ProSense agent in the center of the atherosclerotic lesions, some of it co-localized with resident macrophages confirming that cathepsins are activated in the inflammatory cells and later released from the dead cells into the core of the plaque. The $\alpha_{v} \beta_{3}$ agent was found in advanced atherosclerotic lesions densely distributed in the adventitia and some was also colocalized with macrophages within the core of the lesions reflecting expected development of new vasculature and already discussed expression on the inflammatory cells. FMT measured concentrations of cathepsin agents ProSense and Cat B FAST in apoE ${ }^{-/-}$mice correlated well with the number of macrophages detected in the six different areas of the aortic arch, supporting the flow cytometric and immunohistochemistry data. However, the correlation between the macrophage count and FMT measured concentrations of $\alpha_{v} \beta_{3}$ agent was poor, which was expected giving the high levels of $\alpha_{v} \beta_{3}$ on the other cells types within the plaque.

Co-localization of cathepsins (B, L, S and K) and macrophages in the core of atherosclerotic lesions of $\mathrm{apoE}^{-/-}$mice and human patients has been documented many times [20$24,28,29,31,34,35]$, confirming an important role of these proteases in the progression of atherosclerosis associated inflammation and the development of vulnerable plaques. Activation of newly developed cathepsin agents, ProSense FAST and Cat B FAST and previously studied [34, 35, 37] polymer-based, ProSense, in atherosclerotic lesions of $\mathrm{apoE}^{-/-}$mice can therefore potentially serve as an imaging biomarker of inflammation.

The co-localization of $\alpha_{v} \beta_{3}$ integrin agent with macrophages and its localization in the adventitia agree well with the previously documented role of $\alpha_{v} \beta_{3}$ integrin in the transformation of monocytes and macrophages in the core of atherosclerotic lesions [19, 39-42] and with the role of this integrin in the neoangiogenesis of vulnerable plaques $[26,42,43]$. The $\alpha_{v} \beta_{3}$ integrin agent is therefore, contrary to cathepsin agents, a molecular imaging biomarker of both inflammation and angiogenesis. The poor correlation between the FMT measured $\alpha_{v} \beta_{3}$ agent concentrations and the macrophage counts may indicate binding of this agent to cells other than inflammatory cells within the atherosclerotic lesions. Binding to myeloid cells within the blood may also influence the FMT signal.

Elevated levels of LDL in the blood of apoE ${ }^{-/-}$mice on regular chow and even greater in mice on chow containing elevated levels of fat and/or cholesterol translate into the formation of atherosclerotic lesions in the aortic arch and proximal arteries as early as 3 months after the birth $[44,45$, 47]. The onset of the disease and the size of the lesions varied between individual animals. FMT in vivo measurements of apoE ${ }^{-1-}$ mice fed with a high cholesterol diet found, when compared to C57BLK/6 mice fed with regular chow, elevated levels of the cathepsin probe activation as early as 15-20 weeks after diet initiation. The levels of activation started to grow exponentially after 25 weeks with an increasing variability among individual animals. In a similar set of FMT measurements, the accumulation of $\alpha_{v} \beta_{3}$ agent in atherosclerotic lesion of $\mathrm{apoE}^{-/-}$mice was detected two to five weeks later and then the concentration of the agent was again increasing exponentially. Our data indicate a good correlation between the activation of cathepsin agents and atherosclerosis-associated inflammatory processes in a longitudinal study using these agents which may provide a noninvasive early means of detection of inflammation in developing atherosclerotic lesions of $\mathrm{apoE}^{-/-}$mice. The somewhat delayed onset of the FMT measured $\alpha_{v} \beta_{3}$ agent accumulation might be the result of the later onset of plaque associated angiogenesis. Since both elevated inflammation as well as angiogenesis are characteristics of vulnerable plaques, both cathepsin as well as $\alpha_{v} \beta_{3}$ integrin agents may serve in their in vivo detection.

Ezetimibe, a selective inhibitor of intestinal cholesterol absorption, has been shown to reduce plasma LDL, increase HDL, and inhibit atherosclerosis associated inflammation $[44,45,48]$. We found that all cathepsin agents at some point detect inhibition of inflammation by ezetimibe in prophylactic longitudinal study of apoE $\mathrm{E}^{-/-}$mice fed on high cholesterol diet. However, to our surprise, the new and nonselective fast acting agent ProSense FAST showed a statistically significant difference only at the 12 week time point, while the polymer-based ProSense detected the statistically different inhibition only at the 31-week time point. The most sensitive for the detection of inhibition by ezetimibe was Cat B FAST, finding the statistically significant inhibition at both 21 and 31 weeks. This may indicate that the selectivity rather than the size of the agent, as might be concluded from previous studies [37], is critical for an efficient detection of atherosclerosis-associated inflammation using cathepsin probes. FMT measurements with the $\alpha_{v} \beta_{3}$ integrin selective agent did not detect ezetimibe-induced inhibition of atherosclerosis progression in $\mathrm{apoE}^{-/-}$mice. This is likely a reflection of a broader spectrum of processes involved in the accumulation of $\alpha_{v} \beta_{3}$ agent in the atherosclerotic lesions. Some of these processes may not be affected by the treatment with ezetimibe or they are affected on different time scales. Hence, a more detailed analysis of the functions of these two types of imaging biomarkers may contribute new details on the development of atherosclerotic lesions.

\section{Conclusions}

Here we describe the functional in vivo imaging evaluation of new cathepsin activatable and $\alpha_{v} \beta_{3}$ integrin targeted agents, prepared using requirements for the design of new 
therapeutics. Using FMT, we measured quantitative changes in atherosclerosis-associated inflammation in apoE $\mathrm{E}^{-/-}$mice and compared these to the previously studied polylysinebased cathepsin agent. We have demonstrated the importance of cathepsin agent selectivity and capability of all agents to quantitatively detect longitudinal progress in inflammation and angiogenesis of atherosclerotic lesions. We have further showed that cathepsin agents detect inhibition of inflammation as a result of prophylactic treatment with the anti-inflammatory therapeutic, ezetimibe [44, 45].

\section{Disclosure}

S. Lin, M. Patel, D. Suresch, B. Connolly, M. Klimas, C. Sur, B. Bednar and are employees of Merck \& Co., Inc. B. Bagna, K. Groves, M. Rajopadhye, and J. Peterson are employees of Perkin Elmer.

\section{References}

[1] P. Libby, "Inflammation in atherosclerosis," Nature, vol. 420, no. 6917, pp. 868-874, 2002.

[2] G. K. Hansson, "Inflammation, atherosclerosis, and coronary artery disease," The New England Journal of Medicine, vol. 352, no. 16, pp. 1685-1695, 2005.

[3] G. K. Hansson and P. Libby, "The immune response in atherosclerosis: a double-edged sword," Nature Reviews Immunology, vol. 6, no. 7, pp. 508-519, 2006.

[4] D. J. Rader and A. Daugherty, "Translating molecular discoveries into new therapies for atherosclerosis," Nature, vol. 451, no. 7181, pp. 904-913, 2008.

[5] F. K. Swirski, M. J. Pittet, M. F. Kircher et al., "Monocyte accumulation in mouse atherogenesis is progressive and proportional to extent of disease," Proceedings of the National Academy of Sciences of the United States of America, vol. 103, no. 27, pp. 10340-10345, 2006.

[6] F. K. Swirski, P. Libby, E. Aikawa et al., "Ly-6Chi monocytes dominate hypercholesterolemia-associated monocytosis and give rise to macrophages in atheromata," Journal of Clinical Investigation, vol. 117, no. 1, pp. 195-205, 2007.

[7] K. J. Woollard and F. Geissmann, "Monocytes in atherosclerosis: subsets and functions," Nature Reviews Cardiology, vol. 7, no. 2, pp. 77-86, 2010.

[8] J. Sanz and Z. A. Fayad, "Imaging of atherosclerotic cardiovascular disease," Nature, vol. 451, no. 7181, pp. 953-957, 2008.

[9] F. A. Jaffer, P. Libby, and R. Weissleder, "Optical and multimodality molecular imaging: insights into atherosclerosis," Arteriosclerosis, Thrombosis, and Vascular Biology, vol. 29, no. 7, pp. 1017-1024, 2009.

[10] A. C. Lindsay and R. P. Choudhury, "Form to function: current and future roles for atherosclerosis imaging in drug development," Nature Reviews Drug Discovery, vol. 7, no. 6, pp. 517-529, 2008.

[11] G. W. Stone, A. Maehara, A. J. Lansky et al., "A prospective natural-history study of coronary atherosclerosis," The New England Journal of Medicine, vol. 364, no. 3, pp. 226-235, 2011.

[12] A. J. Sinusas, F. Bengel, M. Nahrendorf et al., "Multimodality cardiovascular molecular imaging, part I," Circulation, vol. 1, no. 3, pp. 244-256, 2008.

[13] M. Nahrendorf, D. E. Sosnovik, B. A. French et al., "Multimodality cardiovascular molecular imaging, Part II," Circulation, vol. 2, no. 1, pp. 56-70, 2009.
[14] C. M. Matter, M. Stuber, and M. Nahrendorf, "Imaging of the unstable plaque: how far have we got?" European Heart Journal, vol. 30, no. 21, pp. 2566-2574, 2009.

[15] J. H. F. Rudd, F. Hyafil, and Z. A. Fayad, "Inflammation imaging in atherosclerosis," Arteriosclerosis, Thrombosis, and Vascular Biology, vol. 29, no. 7, pp. 1009-1016, 2009.

[16] M. M. Sadeghi, D. K. Glover, G. M. Lanza, Z. A. Fayad, and L. L. Johnson, "Imaging atherosclerosis and vulnerable plaque," Journal of Nuclear Medicine, vol. 51, supplement 1, pp. 51S65S, 2010.

[17] D. Vancraeynest, A. Pasquet, V. Roelants, B. L. Gerber, and J. L. J. Vanoverschelde, "Imaging the vulnerable plaque," Journal of the American College of Cardiology, vol. 57, no. 20, pp. 19611979, 2011.

[18] W. Li, X. M. Yuan, A. G. Olsson, and U. T. Brunk, "Uptake of oxidized LDL by macrophages results in partial lysosomal enzyme inactivation and relocation," Arteriosclerosis, Thrombosis, and Vascular Biology, vol. 18, no. 2, pp. 177-184, 1998.

[19] A. S. Antonov, F. D. Kolodgie, D. H. Munn, and R. G. Gerrity, "Regulation of macrophage foam cell formation by $\alpha_{v} \beta_{3}$ integrin: Potential role in human atherosclerosis," American Journal of Pathology, vol. 165, no. 1, pp. 247-258, 2004.

[20] V. Y. Reddy, Q. Y. Zhang, and S. J. Weiss, "Pericellular mobilization of the tissue-destructive cysteine proteinases, cathepsins B, L, and S, by human monocyte-derived macrophages," Proceedings of the National Academy of Sciences of the United States of America, vol. 92, no. 9, pp. 3849-3853, 1995.

[21] G. K. Sukhova, G. P. Shi, D. I. Simon, H. A. Chapman, and P. Libby, "Expression of the elastolytic cathepsins S and $\mathrm{K}$ in human atheroma and regulation of their production in smooth muscle cells," Journal of Clinical Investigation, vol. 102, no. 3, pp. 576-583, 1998.

[22] J. Liu, G. K. Sukhova, J. S. Sun et al., "Lysosomal cysteine proteases in atherosclerosis," Arteriosclerosis, Thrombosis, and Vascular Biology, vol. 24, no. 8, pp. 1359-1366, 2004.

[23] A. Garcia-Touchard, T. D. Henry, G. Sangiorgi et al., "Extracellular proteases in atherosclerosis and restenosis," Arteriosclerosis, Thrombosis, and Vascular Biology, vol. 25, no. 6, pp. 11191127, 2005.

[24] S. P. M. Lutgens, K. B. J. M. Cleutjens, M. J. A. P. Daemen, and S. Heeneman, "Cathepsin cysteine proteases in cardiovascular disease," FASEB Journal, vol. 21, no. 12, pp. 3029-3041, 2007.

[25] G. L. ten Kate, E. J. G. Sijbrands, R. Valkema et al., "Molecular imaging of inflammation and intraplaque vasa vasorum: a step forward to identification of vulnerable plaques?" Journal of Nuclear Cardiology, vol. 17, no. 5, pp. 897-912, 2010.

[26] P. R. Moreno, K. R. Purushothaman, V. Fuster et al., "Plaque neovascularization is increased in ruptured atherosclerotic lesions of human aorta: implications for plaque vulnerability," Circulation, vol. 110, no. 14, pp. 2032-2038, 2004.

[27] R. Weissleder, C. H. Tung, U. Mahmood, and A. Bogdanov, "In vivo imaging of tumors with protease-activated near-infrared fluorescent probes," Nature Biotechnology, vol. 17, no. 4, pp. 375-378, 1999.

[28] S. Fujimoto, D. Hartung, S. Ohshima et al., "Molecular imaging of matrix metalloproteinase in atherosclerotic lesions. Resolution with dietary modification and statin therapy," Journal of the American College of Cardiology, vol. 52, no. 23, pp. 1847-1857, 2008.

[29] J. Chen, C. H. Tung, U. Mahmood et al., "In vivo imaging of proteolytic activity in atherosclerosis," Circulation, vol. 105, no. 23, pp. 2766-2771, 2002. 
[30] F. A. Jaffer, P. Libby, and R. Weissleder, "Molecular imaging of cardiovascular disease," Circulation, vol. 116, no. 9, pp. 10521061, 2007.

[31] F. A. Jaffer, D. E. Kim, L. Quinti et al., "Optical visualization of cathepsin K activity in atherosclerosis with a novel, proteaseactivatable fluorescence sensor," Circulation, vol. 115, no. 17, pp. 2292-2298, 2007.

[32] F. A. Jaffer, C. Vinegoni, M. C. John et al., "Real-time catheter molecular sensing of inflammation in proteolytically active atherosclerosis," Circulation, vol. 118, no. 18, pp. 1802-1809, 2008.

[33] M. A. Calfon, C. Vinegoni, V. Ntziachristos, and F. A. Jaffer, "Intravascular near-infrared fluorescence molecular imaging of atherosclerosis: toward coronary arterial visualization of biologically high-risk plaques," Journal of Biomedical Optics, vol. 15, no. 1, Article ID 011107, 2010.

[34] D. E. Kim, J. Y. Kim, D. Schellingerhout et al., "Molecular imaging of Cathepsin B proteolytic enzyme activity reflects the inflammatory component of atherosclerotic pathology and can quantitatively demonstrate the antiatherosclerotic therapeutic effects of atorvastatin and glucosamine," Molecular Imaging, vol. 8, no. 5, pp. 291-301, 2009.

[35] D. E. Kim, J. Y. Kim, D. Schellingerhout et al., "Protease imaging of human atheromata captures molecular information of atherosclerosis, complementing anatomic imaging," Arteriosclerosis, Thrombosis, and Vascular Biology, vol. 30, no. 3, pp. 449-456, 2010.

[36] F. A. Jaffer, M. A. Calfon, A. Rosenthal et al., "Two-dimensional intravascular near-infrared fluorescence molecular imaging of inflammation in atherosclerosis and stent-induced vascular injury," Journal of the American College of Cardiology, vol. 57, no. 25, pp. 2516-2526, 2011.

[37] M. Nahrendorf, P. Waterman, G. Thurber et al., "Hybrid in vivo FMT-CT imaging of protease activity in atherosclerosis with customized nanosensors," Arteriosclerosis, Thrombosis, and Vascular Biology, vol. 29, no. 10, pp. 1444-1451, 2009.

[38] M. M. Sadeghi, S. Krassilnikova, J. Zhang et al., "Detection of injury-induced vascular remodeling by targeting activated $\alpha_{v} \beta_{3}$ integrin in vivo," Circulation, vol. 110, no. 1, pp. 84-90, 2004.

[39] J. Waldeck, F. Häger, C. Höltke et al., "Fluorescence reflectance imaging of macrophage-rich atherosclerotic plaques using an $\alpha_{v} \beta_{3}$ integrin-targeted fluorochrome," Journal of Nuclear Medicine, vol. 49, no. 11, pp. 1845-1851, 2008.

[40] P. M. Winter, A. M. Morawski, S. D. Caruthers et al., "Molecular imaging of angiogenesis in early-stage atherosclerosis with $\alpha_{v} \beta_{3}$-integrin-targeted nanoparticles," Circulation, vol. 108, no. 18 , pp. 2270-2274, 2003.

[41] I. Laitinen, A. Saraste, E. Weidl et al., "Evaluation of $\alpha_{v} \beta_{3}$ integrin-targeted positron emission tomography tracer ${ }^{18} \mathrm{~F}$ galacto-RGD for imaging of vascular inflammation in atherosclerotic mice," Circulation, vol. 2, no. 4, pp. 331-338, 2009.

[42] J. Heroux, A. M. Gharib, N. S. Danthi, S. Cecchini, J. Ohayon, and R. I. Pettigrew, "High-affinity $\alpha_{v} \beta_{3}$ integrin targeted optical probe as a new imaging biomarker for early atherosclerosis: initial studies in watanabe rabbits," Molecular Imaging and Biology, vol. 12, no. 1, pp. 2-8, 2010.

[43] C. Burtea, S. Laurent, O. Murariu et al., "Molecular imaging of $\alpha_{v} \beta_{3}$ integrin expression in atherosclerotic plaques with a mimetic of RGD peptide grafted to Gd-DTPA," Cardiovascular Research, vol. 78, no. 1, pp. 148-157, 2008.

[44] P. J. Kuhlencordt, P. Padmapriya, S. Rützel et al., "Ezetimibe potently reduces vascular inflammation and arteriosclerosis in
eNOS-deficient ApoE ko mice," Atherosclerosis, vol. 202, no. 1, pp. 48-57, 2009.

[45] K. Graf, T. Dietrich, M. Tachezy et al., "Monitoring therapeutical intervention with ezetimibe using targeted near-infrared fluorescence imaging in experimental atherosclerosis," Molecular Imaging, vol. 7, no. 2, pp. 68-76, 2008.

[46] S. Kossodo, M. Pickarski, S. A. Lin et al., "Dual in vivo quantification of integrin-targeted and protease-activated agents in cancer using fluorescence molecular tomography (FMT)," Molecular Imaging and Biology, vol. 12, no. 5, pp. 488-499, 2010.

[47] S. H. Zhang, R. L. Reddick, J. A. Piedrahita, and N. Maeda, "Spontaneous hypercholesterolemia and arterial lesions in mice lacking apolipoprotein E," Science, vol. 258, no. 5081, pp. 468-471, 1992.

[48] T. Dietrich, T. Hucko, R. Bourayou et al., "High resolution magnetic resonance imaging in atherosclerotic mice treated with ezetimibe," International Journal of Cardiovascular Imaging, vol. 25, no. 8, pp. 827-836, 2009. 


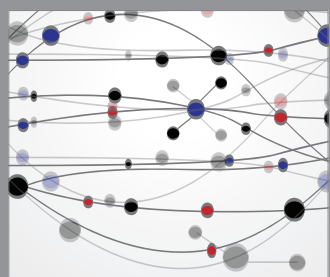

The Scientific World Journal
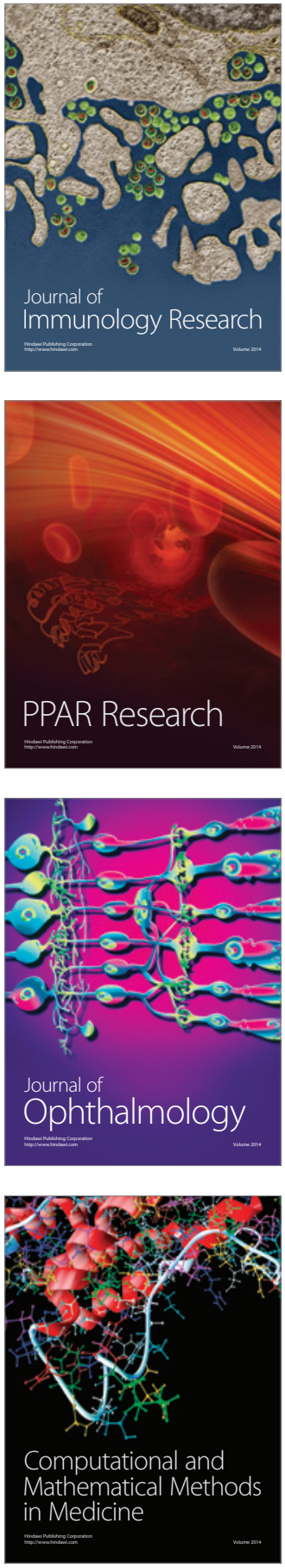

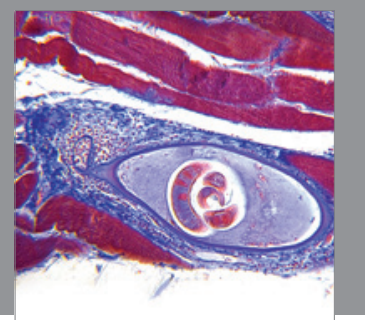

Gastroenterology

Research and Practice
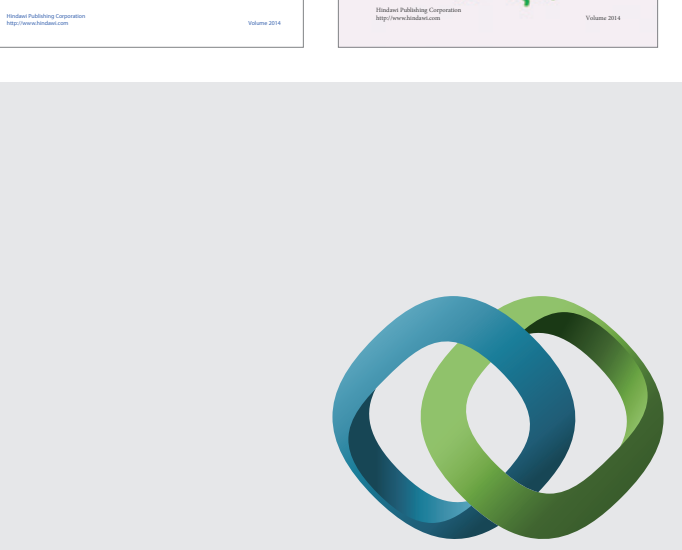

\section{Hindawi}

Submit your manuscripts at

http://www.hindawi.com
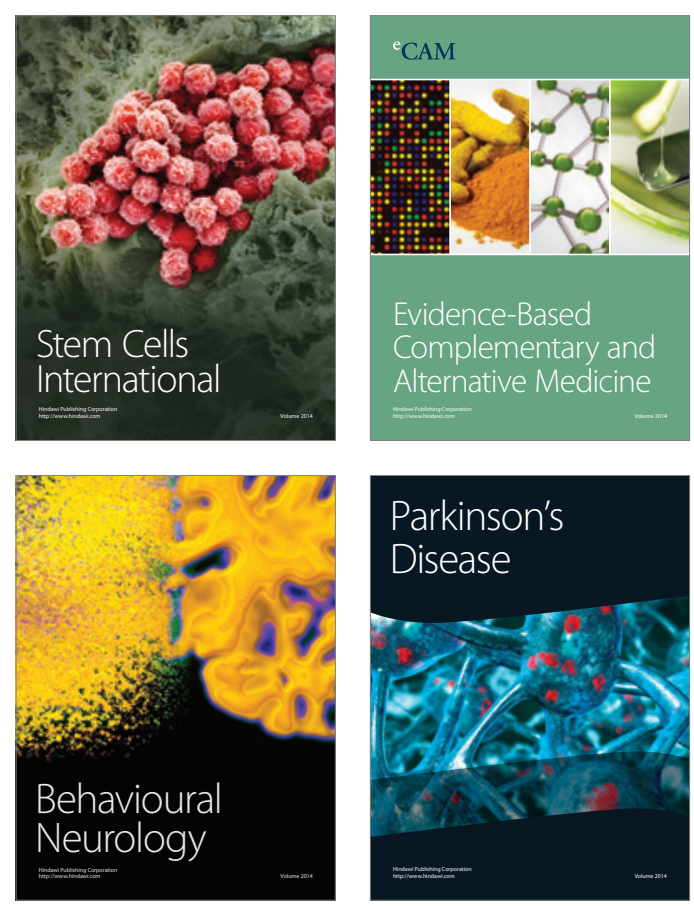

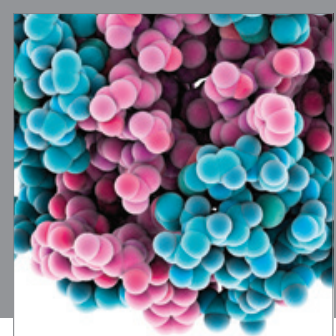

Journal of
Diabetes Research

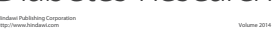

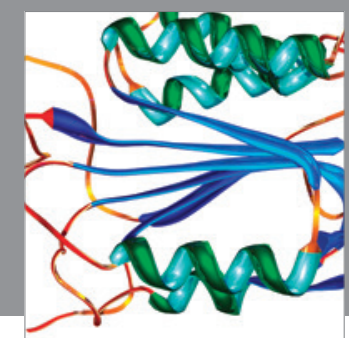

Disease Markers
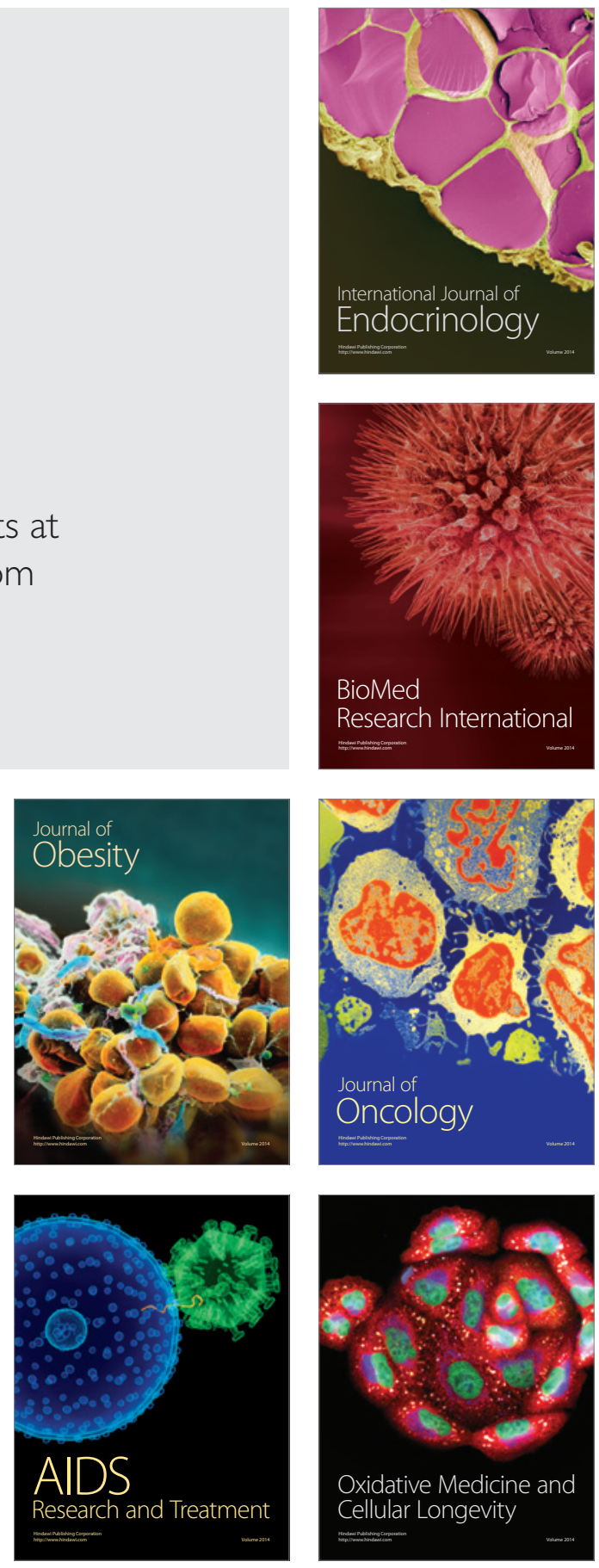